\title{
Thermal transport at (001) twist grain boundaries in $\mathrm{UO}_{2}$
}

\author{
B. Deng (邓博文) $)^{1}$, A. Chernatynskiy ${ }^{2}$, S. B. Sinnott ${ }^{3}$ and S. R. Phillpot* \\ Department of Materials Science and Engineering \\ University of Florida \\ Gainesville FL 32611
}

To be submitted to Journal of Nuclear Materials

\begin{abstract}
We use phonon wave-packet dynamics to study the phonon transmission at $\mathrm{UO}_{2}$ twist grain boundaries as a function of phonon frequency, mode and incident angle. We find that grain boundaries with more disordered structures show stronger phonon transmission than more ordered grain boundaries. The transmission coefficients are used to calculate the grain boundary thermal conductance at elevated temperature.
\end{abstract}

*Corresponding author: sphil@mse.ufl.edu

${ }^{1}$ Present Address: Department of Computer Science, Montana Tech of the University of Montana, Butte, MT 59701

${ }^{2}$ Present Address: Department of Physics, Missouri University of Science and Technology, Rolla MO 65409

${ }^{3}$ Present Address: Department of Materials Science and Engineering, Pennsylvania State University, State College PA 16802 


\section{INTRODUCTION}

The thermal conductivity is a key performance metric for uranium dioxide $\left(\mathrm{UO}_{2}\right)$ nuclear fuel. Under the extreme operating conditions of high pressure and high temperature in a nuclear reactor, the fuel material undergoes various changes, both physical and chemical. Of particular interest for this work is the evolution of the microstructure, including point defects, dislocations and grain boundaries. These defects are present in the microstructure at the beginning of life; during burnup more defects are created, they evolve and some are destroyed. All of these defects impede the phonon-mediated heat transport, the main heat conduction mechanism in these insulating oxide fuels. Grain boundaries (GBs), among the most prevalent of these defects, break the periodicity of the crystal, and are often characterized by significant amounts of atomic disorder.

The obstruction of heat transport at a GB leads to a jump in temperature across the boundary, from which the interfacial thermal resistance, or Kapitza resistance, is defined. The Kapitza resistance plays a critical role in the transport of thermal energy, not only in nuclear fuel, but in many other areas, such as thermal barrier coatings [1], thermoelectrics [2] and nanoelectronics [3]. Swartz and Pohl [4] discussed two theoretical models for phonon-mediated interfacial thermal transport, the acoustic mismatch model (AMM) and the diffuse mismatch model (DMM). The AMM model takes a continuum acoustics approach, in which the interfacial phonon transmission is determined by the mismatch in the acoustic impedance across the interface. For the GB, however, the AMM predicts that there is no interfacial resistance, since the material on the two sides of the interface is the same, and thus there is no acoustic mismatch. By contrast, the DMM model assumes all phonons are diffusely scattered at the interface; that is, their transmission is solely determined by the densities of phonon states on both sides of the interface. Correspondingly, the DMM will predict that all twist GB should have the same Kapitza resistance, which is not the case. While each model appears to have a range of applicability, neither offers a comprehensive understanding of interfacial thermal transport. A significant aspect of both models is they completely neglect the detailed atomic structure of an interface. There is, however, extensive evidence, both from experiment [5] and simulation [5], that the structure of interfaces is important in many materials. Non-equilibrium molecular 
dynamics (NEMD) methods [6] and phonon wave-packet dynamics (PWD) methods [7] do include the full consideration of the atomic structure of an actual interface, and can thus offer a more nuanced description of interfacial thermal transport than that offered by the DMM and AMM alone.

There have been several previous studies of interfacial thermal resistance in $\mathrm{UO}_{2}$ grain boundaries using the NEMD method. Watanabe et al. [8] extracted the average Kapitza resistance of an ensemble of rather similar $\mathrm{UO}_{2}$ tilt grain boundaries by carrying out simulations on a model of a textured $\mathrm{UO}_{2}$ polycrystal. Recently, Chen et al. [9] explored the Kapitza resistance of individual crystallographically well-defined $\mathrm{UO}_{2}$ grain boundaries. They computed the Kapitza resistance of various $\mathrm{UO}_{2}$ tilt grain boundaries by using direct $\mathrm{MD}$ simulations and found that the Kapitza resistance increases essentially linearly with the grain boundary energy; the same relationship had previously been seen for grain boundaries in diamond [6], suggesting that this may be a rather general result. This seems physically reasonable, because the GB energy is largely a measure of the structural disorder. However, such direct simulation of the thermal conductivity does not provide any detailed information on phonon scattering mechanisms. The phonon wave-packet dynamics (PWD) method, by contrast, is able to provide such information about phonon-interface scatterings [10]. Previously, Schelling et al. [10,11] have used the PWD method to characterize the phonon scattering processes associated with the interfacial resistance of Si grain boundaries; here we apply it to grain boundaries in $\mathrm{UO}_{2}$. In the present work, we use the PWD method to study the phonon transmission coefficients of phonons at two (001) twist grain boundaries and how their detailed atomic structures affect the transmission.

\section{MODEL PREPARATION}

According to experimental structural analysis on a depleted $\mathrm{UO}_{2}$ fuel sample [12], $16 \%$ percent of the boundaries can be categorized as coincident site lattice (CSL) boundaries in which the small $\Sigma$ value grain boundaries are abundant. In this work, the (001) $\Sigma 5$ and $\Sigma 17$ twist grain boundaries, with rotation angles of $\Theta=36.86^{\circ}$ and $28.07^{\circ}$ respectively, are studied. Both 
structures have similar size in the cross-sectional plane, being $24.46 \AA$ × $24.46 \AA$ (a $2 \times 2$ planar unit cell of $\Sigma 5$ ) and $22.54 \AA$ X $22.54 \AA$ (a 1 x 1 planar unit cell of $\Sigma 17$ ).

The top views of the crystal structure around both the grain boundary models are shown in Figure 1. The simulation cells have a much larger dimension in the direction perpendicular to the grain boundary plane (about $4300 \AA, 800$ unit cells). This is to ensure that the wave packet can be properly localized and the entirety of the phonon-interface scattering event can be tracked.

The interatomic interactions are described using the Busker interatomic potential [13-15]. We have previously used the direct MD method with the same potential to investigate the thermal conductivity of pure $\mathrm{UO}_{2}[8]$, as well as the effects of point defects [16] and dislocations [17] on thermal conductivity. The Busker potential has also been used to extract the thermal conductivity at $\mathrm{UO}_{2}$ grain boundary from a polycrystalline model [8]. Previously, Nerikar et al. also used both the Busker and Basak potential to study the segregation energy of Xe at $\Sigma 5$ tilt $\mathrm{UO}_{2}$ grain boundary using the same potential $[18,19]$. The grain boundary energy of our $\Sigma 5$ twist grain boundary model is $0.13 \mathrm{eV} / \AA^{2}$, which is comparable with that of the $\mathrm{UO}_{2} \Sigma 5$ tilt grain boundary[12].
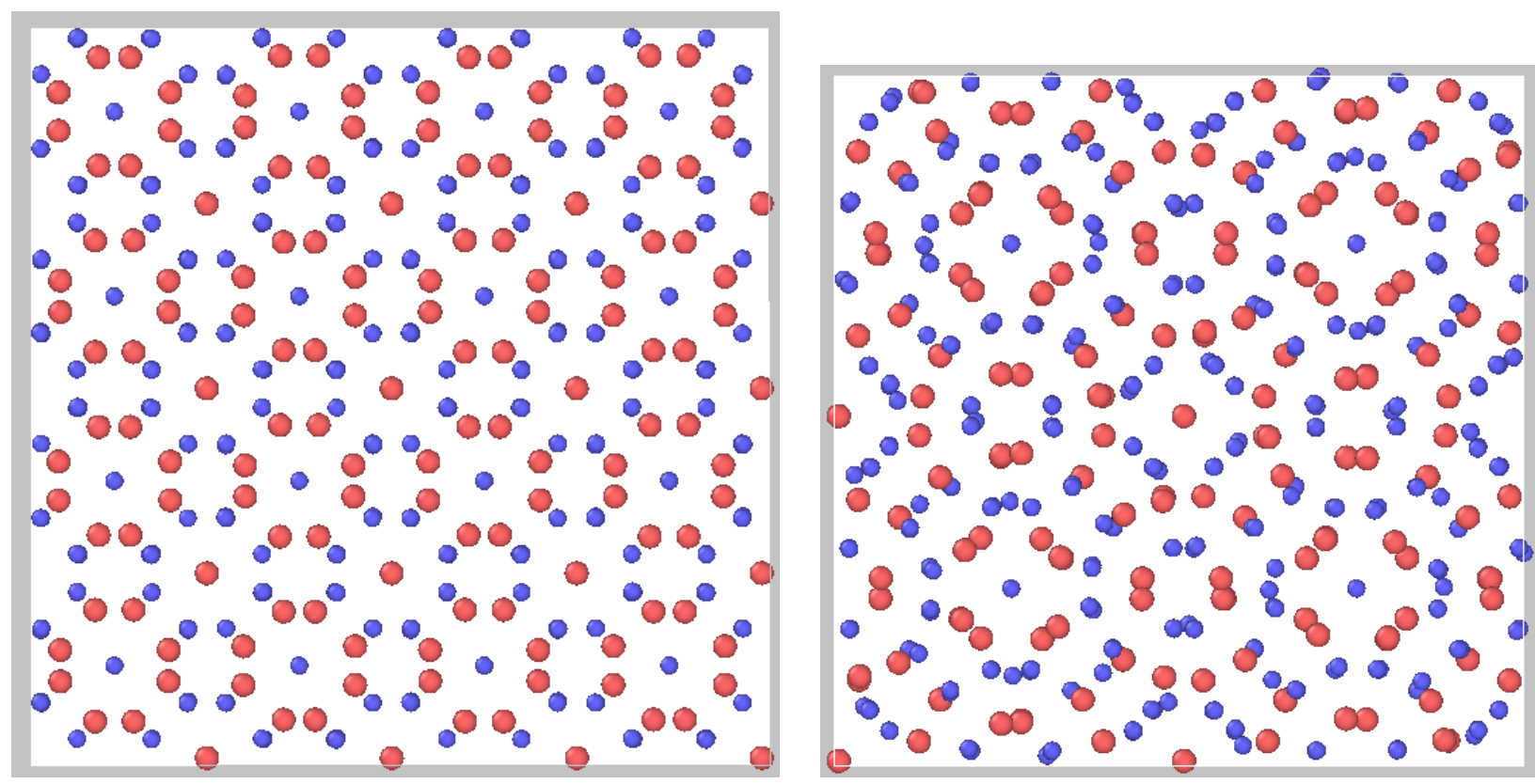
Figure 1 Top view [001] of the crystal structure of $\Sigma 5$ (left) and $\Sigma 17$ (right) twist grain boundary. Red and blue atoms denote uranium and oxygen respectively.

The initial structures, generated purely from crystallographic analysis, are annealed at 2000 $\mathrm{K}$ for $60 \mathrm{ps}$, after which the structures are quenched such that the forces on every ion are small. A very rigorous final relaxation process has to be performed for the structures used in the PWD simulations, so that all forces on atoms are very small, less than $10^{-8} \mathrm{eV} / \AA . \AA$. This eliminates any excess potential energy in the system that can swamp the signal obtained from the low-energy wave-packet.

\section{PHONON SCATTERINGS FROM WAVE-PACKET DYNAMICS}

\section{A. Basics of phonon wave-packets}

The phonon wave-packet dynamics method is based on wavelet transforms [20] and molecular dynamics simulation. The basic idea of PWD is to form a phonon wave packet so that it is localized in both real space and reciprocal space. We generate our wave packet $[21,22]$ with the phonon propagation direction along $z[001]$, that is normal to the GB plane. The initial atomic displacement (with maximum amplitude of $10^{-5} \AA$ ) is generated as a Gaussian wave packet:

$$
\mathbf{u}_{i l}(t=0)=\frac{A}{\sqrt{m_{i}}} \boldsymbol{\varepsilon}_{i \lambda \mathbf{k}_{0}} \exp \left[i \mathbf{k}_{0} \cdot\left(\mathbf{R}_{l}-\mathbf{R}_{0}\right)\right] \exp \left[-\frac{\left(\mathbf{R}_{l}-\mathbf{R}_{0}\right)^{2}}{\eta^{2}}\right]
$$

where A controls the amplitude of the wave-packet and $\eta$ controls the width of the wave-packet; $\mathbf{u}_{i l}$ denotes the atomic displacement of the $\mathrm{i}$-th atom in the $\mathrm{l}$-th primitive cell, $\mathrm{N}$ is the total number of primitive cells, $m_{i}$ is the mass of the i-th atom, $\mathbf{R}_{l}$ is the coordinates of the $l$-th cell, the $a_{\lambda \mathbf{k}}$ is the normal coordinates of the phonon with wave vector $\mathbf{k}$ and branch $\lambda, \omega_{\lambda \mathbf{k}}$ is the phonon frequency, $\boldsymbol{\varepsilon}_{i \lambda \mathbf{k}}$ and $\boldsymbol{\varepsilon}_{i \lambda \mathbf{k}}^{*}$ are the eigenvector and its complex conjugate of the corresponding phonon mode for the $\mathrm{i}$-th atom respectively and $\mathrm{t}$ is the simulation time. 
To obtain the velocity for each atom as the initial conditions for an MD simulation, a subsequent inverse discrete Fourier transformation is performed to obtain the normal coordinates $a_{\lambda \mathbf{k}}$ :

$$
a_{\lambda \mathbf{k}}=\sum_{i l} \sqrt{\frac{m_{i}}{N}} \mathbf{u}_{i l} \cdot \boldsymbol{\varepsilon}_{i \lambda \mathbf{k}}^{*} \exp \left(-i \mathbf{k} \cdot \mathbf{R}_{l}\right)
$$

The atomic displacement can be rewritten as a summation of all the normal modes:

$$
\mathbf{u}_{i l}(t)=\frac{1}{\sqrt{N m_{i}}} \sum_{\lambda \mathbf{k}} a_{\lambda \mathbf{k}} \boldsymbol{\varepsilon}_{i \lambda \mathbf{k}} \exp \left(i \mathbf{k} \cdot \mathbf{R}_{l}-i \omega_{\lambda \mathbf{k}} t\right)
$$

Consequently the atomic velocity, $\mathbf{v}_{i l}$, can then be computed as a time derivative of the atomic displacements:

$$
\mathbf{v}_{i l}(t)=\frac{1}{\sqrt{N m_{i}}} \sum_{\lambda \mathbf{k}} a_{\lambda \mathbf{k}} \boldsymbol{\varepsilon}_{i \lambda \mathbf{k}} \exp \left(i \mathbf{k} \cdot \mathbf{R}_{l}-i \omega_{\lambda \mathbf{k}} t\right) i \omega_{\lambda \mathbf{k}}
$$

After launching the wave packet towards the grain boundary, scattering events take place, with part of the wave packet transmitting through and part reflecting back. By determining the energy associated with the transmission and reflection, we calculate the phonon transmission at the grain boundary, and thus calculate the Kapitza conductance by integration over the first Brillouin Zone (BZ):

$$
\sigma=\frac{1}{(2 \pi)^{2}} \int_{\mathbf{k}} \sum_{\lambda}^{+} \hbar \omega_{\lambda \mathbf{k}} v_{z, \lambda \mathbf{k}} \alpha_{\lambda \mathbf{k}} \frac{d n(\omega, T)}{d T} d \mathbf{k}
$$

where $\alpha_{\lambda \mathbf{k}}$ is the phonon transmission coefficient determined from the PWD simulations, $\hbar$ is the reduced Planck constant and $n(\omega, T)$ is the Bose-Einstein distribution function at temperature T. When performing the calculation of the conductance from Eq.(5), the integration over the first BZ is converted into a summation over the grid of sampling k-points. This equation is able to provide the Kapitza conductance based on the phonon energy transmission coefficient calculated from the PWD method.

For the PWD method, due to the very small amplitude of the incoming wave packets, only elastic scattering is captured, thus the frequencies of incoming and outgoing phonons are 
always the same. Inelastic scattering, which is not captured in the wave-packet simulation, would produce phonons of different frequencies due to the phonon-phonon interactions and would correspondingly increase the conductance with temperature, as observed in several NEMD simulations. A determination of these anharmonic effects would require the investigation of how the transmission probability of incoming wave-packet at elevated temperatures. However, it would be very difficult to pick out the signal of wave-packet against the background of the thermal vibrations.

\section{B. Phonon wave-packet transmission at $\Sigma 5$ twist grain boundary}

We start by applying the PWD method to the $\Sigma 5$ twist grain boundary. Phonon wave packets centered on different vibrational modes and frequencies are generated and launched toward the grain boundary. The contributions to the boundary conductance from various normally incident phonon modes is summarized in Table 1. To more fully understand the phonon scattering process, the cases of LA and LO modes will be analyzed below.

Table 1 Mode wise contribution to boundary conductance.

\begin{tabular}{ccccc}
\hline Modes & LA & TA & LO & TO \\
\hline Percentage $(\%)$ & 47 & 27 & 19 & 7 \\
\hline
\end{tabular}

The transmission coefficients of the LA and LO incident wave packets are shown as the dashed lines in Figure 2. It is clear that for the LA mode (blue squares) that there is a strong dependence of the transmission coefficient on incident phonon frequency. In particular, at low frequencies, corresponding to long wavelengths, the transmission is nearly $100 \%$. This high transmission coefficient arises because the thickness of the grain boundary along the phonon propagation direction is much smaller than the wavelength of the phonon, providing very little opportunity for their interaction. Such high transmission coefficients for low frequency LA modes have been seen for a number of other systems, [23-26] and are consistent with the AMM model. However, as the phonon frequency increases, the transmission coefficient gradually drops to a very low value. To better illustrate the different scattering scenarios of low and high frequency phonons, Figure 3 shows a series of snapshots of two different phonon 
wave packets propagating through the grain boundary. The low frequency case in Figure $3(A)$ is the $0.63 \mathrm{THz}$ LA wave packet, corresponding to an average wavelength about $109 \AA$, which is much greater than the dimension of the grain boundary. The phonon wave packet passes through the GB (denoted by the vertical dashed-line in the center of each panel) essentially unscattered. This is consistent with the transmission coefficients in Figure 2, which shows that transmission coefficient for this phonon wave packet is close to unity. This phenomenon is also expected from the AMM model, as at low frequencies, the continuum assumption could be applied. The high frequency cases is a $4.19 \mathrm{THz}$ LA wave packet with average wavelength of about $15.6 \AA$ A. Here, as shown in Figure 3(B), there is strong scattering of the wave packet at the $\mathrm{GB}$, with part of the wave packet being reflected back. The transmitted and reflected wave packets closer to the grain boundary in Figure 2(B) were emitted from the GB long after the initial scattering event. That is, the energy that they carry was stored as vibrational excitations in the $G B$, for an extended period of time. Thus the symmetry between these reflected and transmitted components is consistent with DMM model, in which all phonons are diffusely scattered, and the transmission probability is determined by the density of phonon states at both sides of the grain boundary. Thus in this case, the transmission probability should be 0.5 from the DMM model. Moreover, there is significant mode coupling, with the generation of transverse phonons, as shown in red, which indicates atomic displacements along the $\mathrm{x}$ and $\mathrm{y}$ directions, i.e., components parallel to the grain boundary. However, the frequency of these phonons does not change, since only elastic phonon scatterings have been captured.

A similar analysis of LO modes is shown in Figure 4(A) shows a $6.38 \mathrm{THz}$ LO incident phonon, for which the transmission coefficient is the highest among the LO modes. Its evolution is very similar to the case discussed above, Figure 3(A). Figure 4(B) shows an extreme case as a contrast. Here a high frequency LO phonon $(\mathrm{f}=16.66 \mathrm{THz})$ is launched toward the grain boundary, and as illustrated in the snapshots, no transmission takes place, with the wave packet being reflected completely. The small group velocity is responsible for the low phonon transmission, as the LO dispersion is flat at both the center and edge of the Brillouin zone. 
To develop a more detailed understanding of the contributions from different vibrational modes to the energy transmission, normal mode analysis is applied to the transmitted and reflected wave packet. For the LA mode, when the frequency is less than $3 \mathrm{THz}$, the wave packet is hardly scattered by the grain boundary at all. When the incident LA frequency is between 3 $\mathrm{THz}$ and $6.7 \mathrm{THz}$, a small amount of the energy is scattered into TA modes, as was clearly identified in Figure 3(B). From 6.7 THz up to the maximum frequency of LA mode, the phonon is scattered into TO modes since TA modes are no longer available. However, mode conversion only contributes a relatively small fraction of the transmitted energy for all LA modes, with the amount of energy transmitted due to the mode conversion almost the same as the reflected amount, suggesting typical DMM behavior. From this, the snapshots of Figure 3(B) can be better understood: a large fraction of the wave packet is scattered as soon as it interacts with the GB, during which the original phonon vibrational mode is preserved. A remaining small fraction of the incident wave packet excites other vibrational modes in the $G B$, which then re-emit equally to the left and right grains. However, for the case of the LO mode, when the frequency is above $8 \mathrm{THz}$, the majority of the energy transmission comes from modes other than the initial wave packet, indicating much stronger GB scattering.

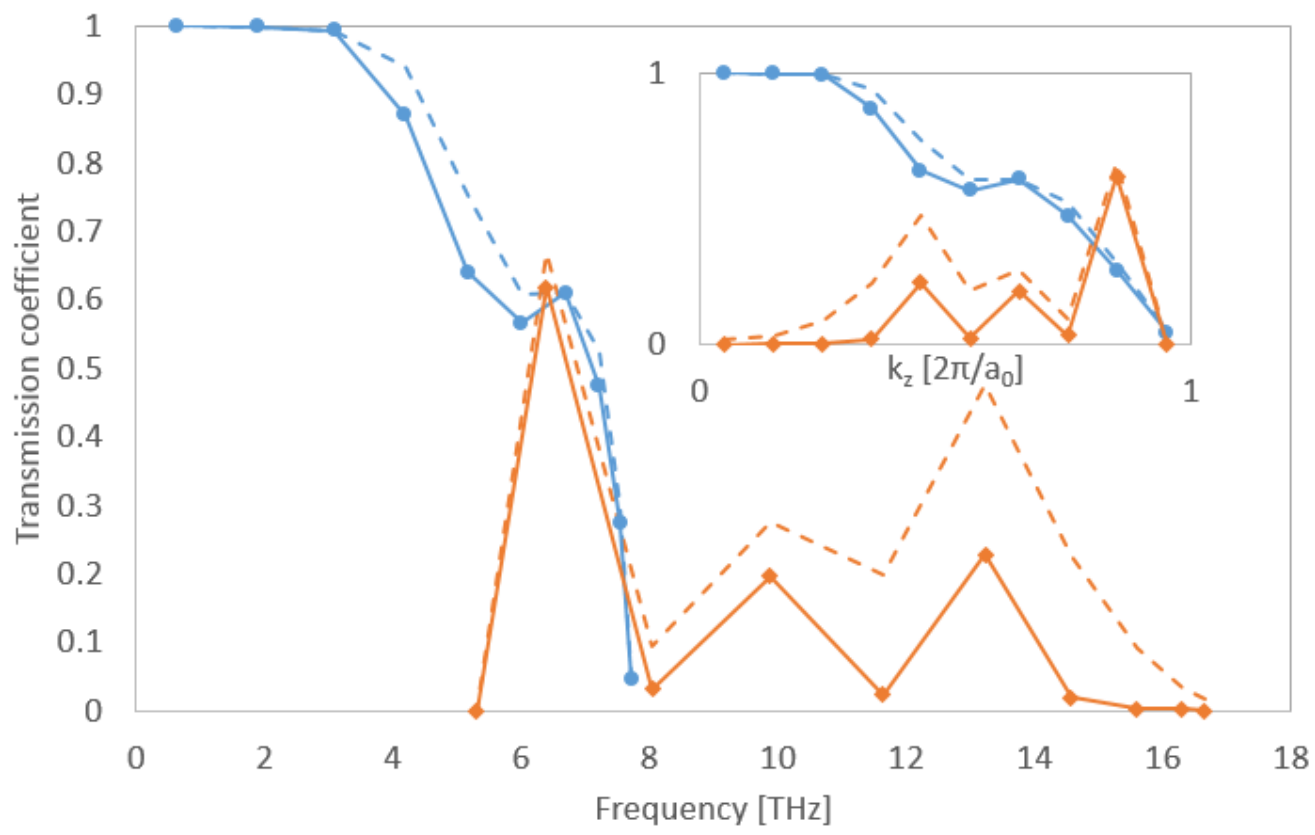

Figure 2 Phonon energy transmission coefficient (LA and LO) for $\Sigma 5$ twist grain boundary as a function of phonon frequency. Blue dots denote the LA mode, while orange diamonds denote the 
LO mode. The dashed lines are the total transmission coefficient, while the solid lines are the contribution of the un-scattered mode. The inset shows the same data in wavevector space.
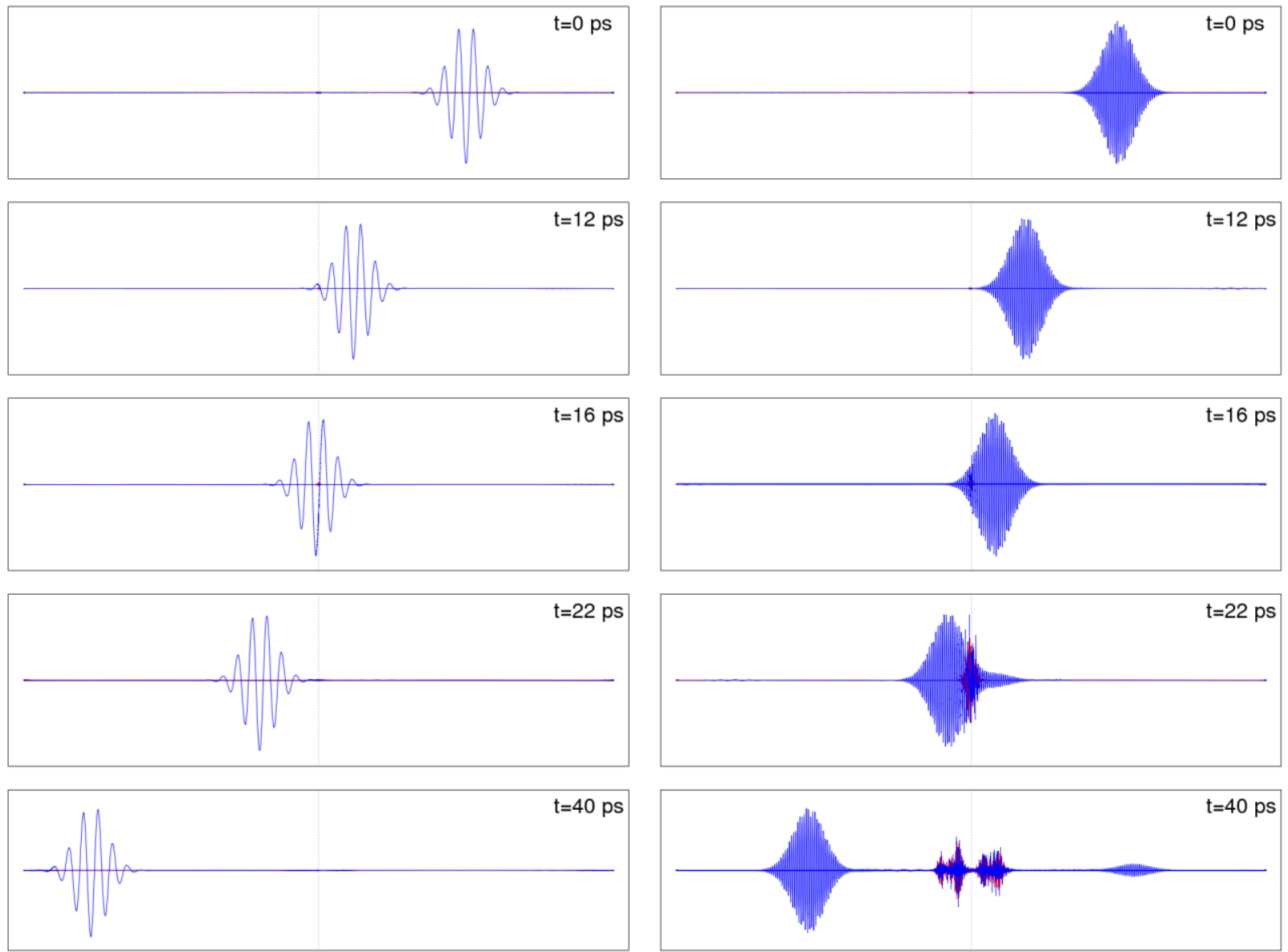

(a)

(b)

Figure 3 Time evolution of atomic displacement for an LA incident phonon wave packet on a $\Sigma 5$ twist grain boundary. The GB is indicated by the dashed line. A) incident frequency of $0.63 \mathrm{THz} ; B$ ) 4.19 $\mathrm{THz}$. 

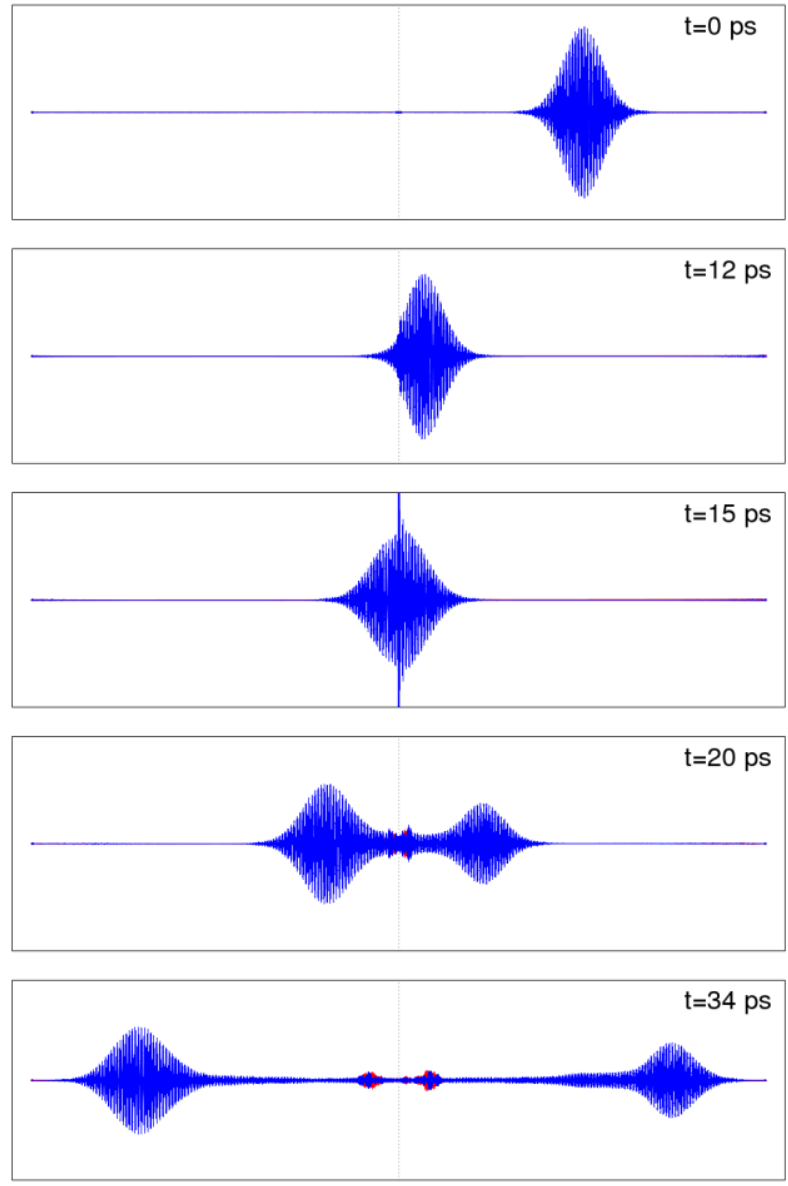

(a)
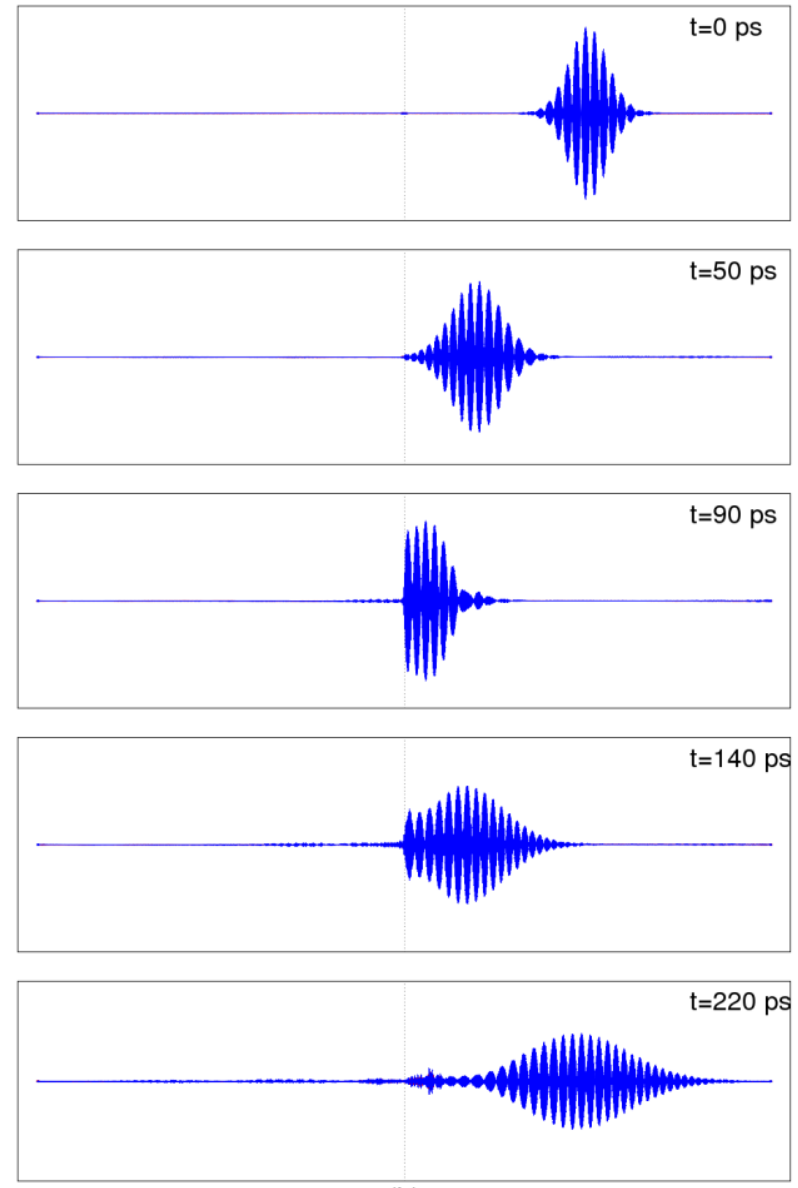

(b)

Figure 4 Time evolution of atomic displacement with LO incident phonon wave packets on a 55 twist grain boundary. The GB is indicated by the dashed line. A) Incident frequency of $6.38 \mathrm{THz} ; \mathrm{B}$ ) 16.66 THz frequency.

In addition to the normal-incident phonon modes, we have also performed simulations for non-normal incident phonons, extensively sampling non-normal LA and LO modes. As shown in Figure $5(b)$, as the component of $k_{x}$ or $k_{y}$ in the wavevector increases, the energy transmission coefficient decreases sharply. The non-uniform decrease appear be due to the detailed atomic structure, as we discuss below. However, such a dependence is not observed in the LO phonon case. We speculate that this is because the phonon frequencies for LO modes are higher (i.e., the wavelengths are shorter) than those for LA modes; thus the LO phonons interact more strongly with the GB atomic structure and the scattering is more diffusive. 

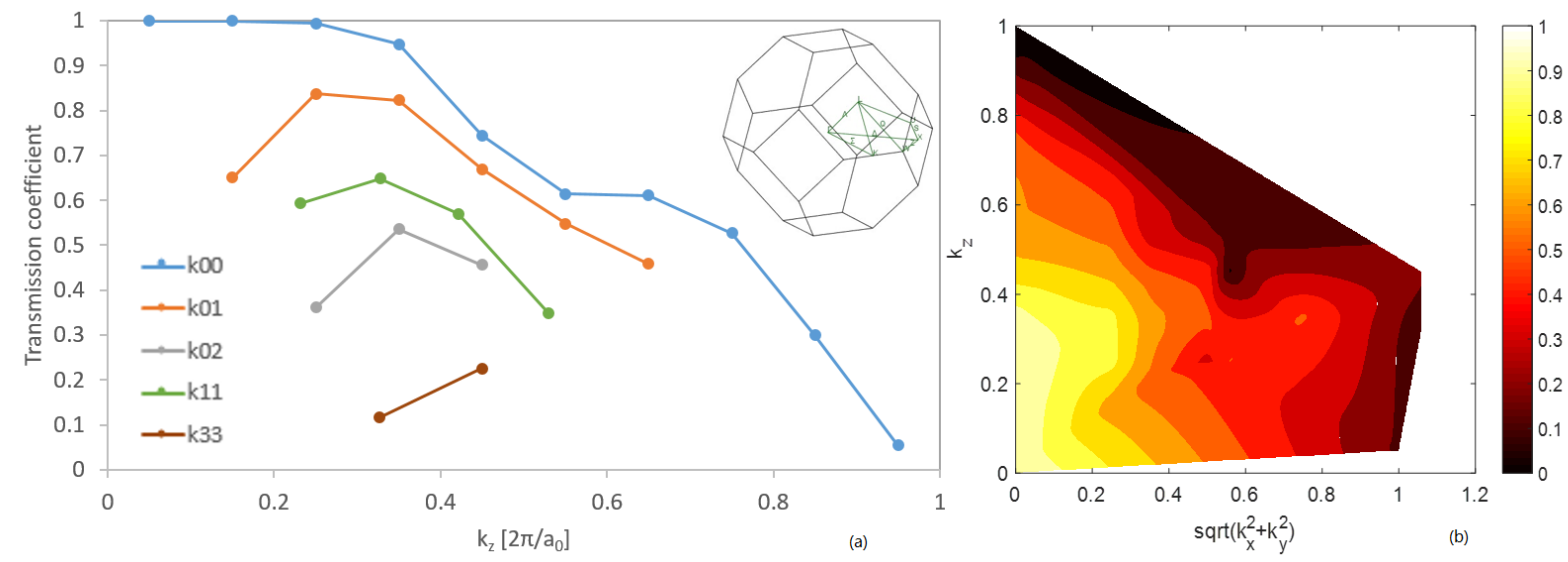

Figure 5 LA energy transmission for various $k_{x}$ and $k_{y}$. The limited range for some branches are due to the edge of first Brillouin Zone and small phonon group velocity. The inset shows the first Brillouin zone as illustration. (b) is a contour plot of the transmission coefficient, from (a).

C. Effects of grain boundary atomic structure

For comparison, we have also performed phonon wave-packet simulations for normal incident LA and LO modes for at a $\Sigma 17$ twist grain boundary, and calculated the corresponding phonon transmission coefficients. As shown in Figure 6, the transmission coefficient of the LA mode of $\Sigma 5$ drops off more steeply with increasing frequency than that of $\Sigma 17$; The LO transmission for $\Sigma 5$ shows some sharp peaks, whereas that for the $\Sigma 17 \mathrm{~GB}$ does not. It seems that the difference must originate from the differences in the atomic structures at the two grain boundaries, because the structures are the same along the direction of phonon transmission in the bulk for the two grain boundary structures. Consequently, we analyze the atomic structures at the grain boundary by determining the inter-plane pair distribution function (PDF) of the atoms in the GB plane, in which only neighboring atoms, out of the grain boundary plane are considered. We find that the PDF of $\Sigma 5$ grain boundary features strong peaks, which reflects the crystal structure of the bulk. However, the PDF of the $\Sigma 17$ GB has much weaker peaks, characteristic of a structure that does not have strong crystalline order. Accordingly, our results are consistent with the idea that the disordered interface structure actually facilitates the LA phonon transmission at larger wavevectors. In order to test this hypothesis, we manually introduce disorder into the originally more ordered $\Sigma 5$ grain boundary by creating various 
numbers of Schottky pairs right at the grain boundary planes. The defected $\Sigma 5$ grain boundary structures are noted by the subscripts "- $n$ " where $n$ is the number of defects introduced. All these structures were annealed at $2000 \mathrm{~K}$ to achieve optimized atomic configurations. Figure 7 shows the PDF of defected $\Sigma 5_{-4}$, which is much less peaked than that of the $\Sigma 5 \mathrm{~GB}$, and more similar to the 517 ; we take this an indication that these two GBs have a similar degree of structural disorder. The phonon transmission coefficients for $\Sigma 5, \Sigma 5_{-n}(n=4,8)$ and $\Sigma 17$ are shown in Figure 8. The LA phonons shows smaller transmission at all the disordered $\Sigma 5$ grain boundaries at low frequencies, but larger transmission at higher frequency; that is the frequency dependence become less strong. For the LO phonons, the transmission coefficient of the $\mathrm{n}=4$ structure is smoother than that of the $\Sigma 5$ and much more closely resembles that of the 217. A similar phonon transmission enhancement due to the disordered interface has been observed in an Si/Ge interface previously [27]. As suggested by the PDF in Figure 7, the disordered atomic structures at the grain boundary has smoothed out the abrupt change of acoustic impedance across the interface.

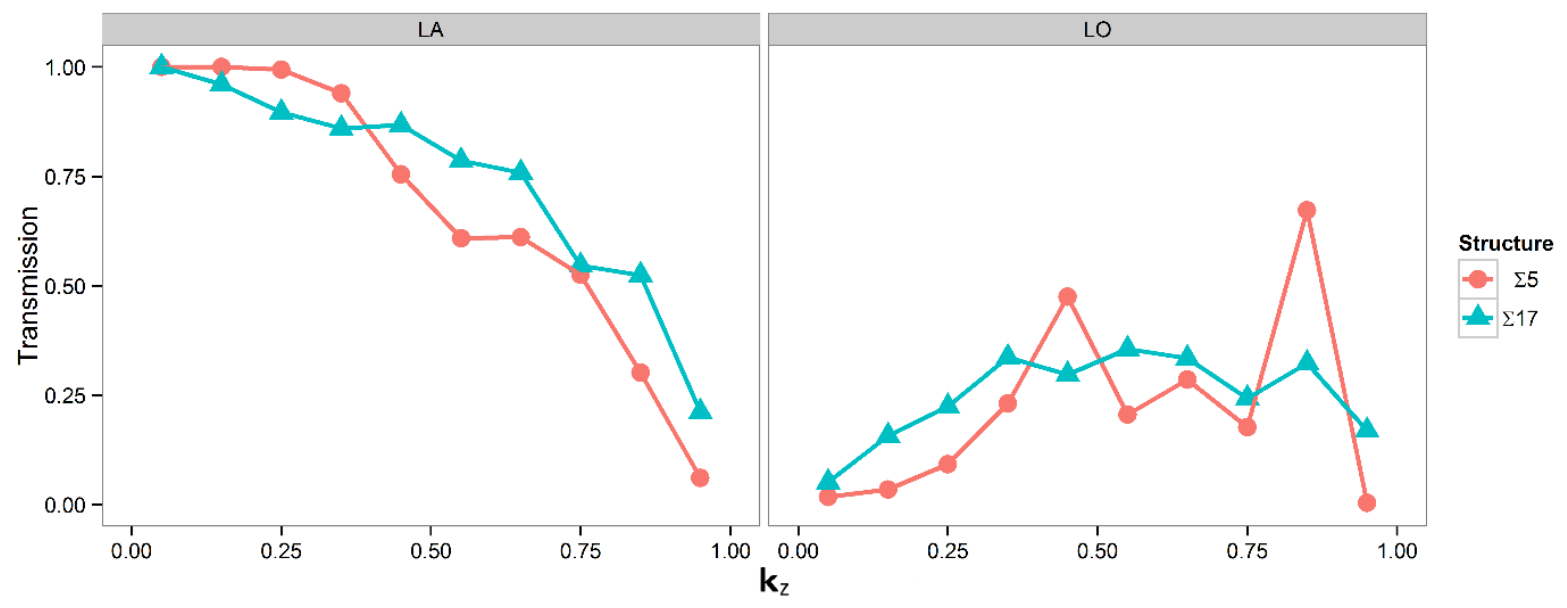

Figure 6 Comparison of LA and LO phonon transmission for $\Sigma 5$ and $\Sigma 17$ grain boundary. 


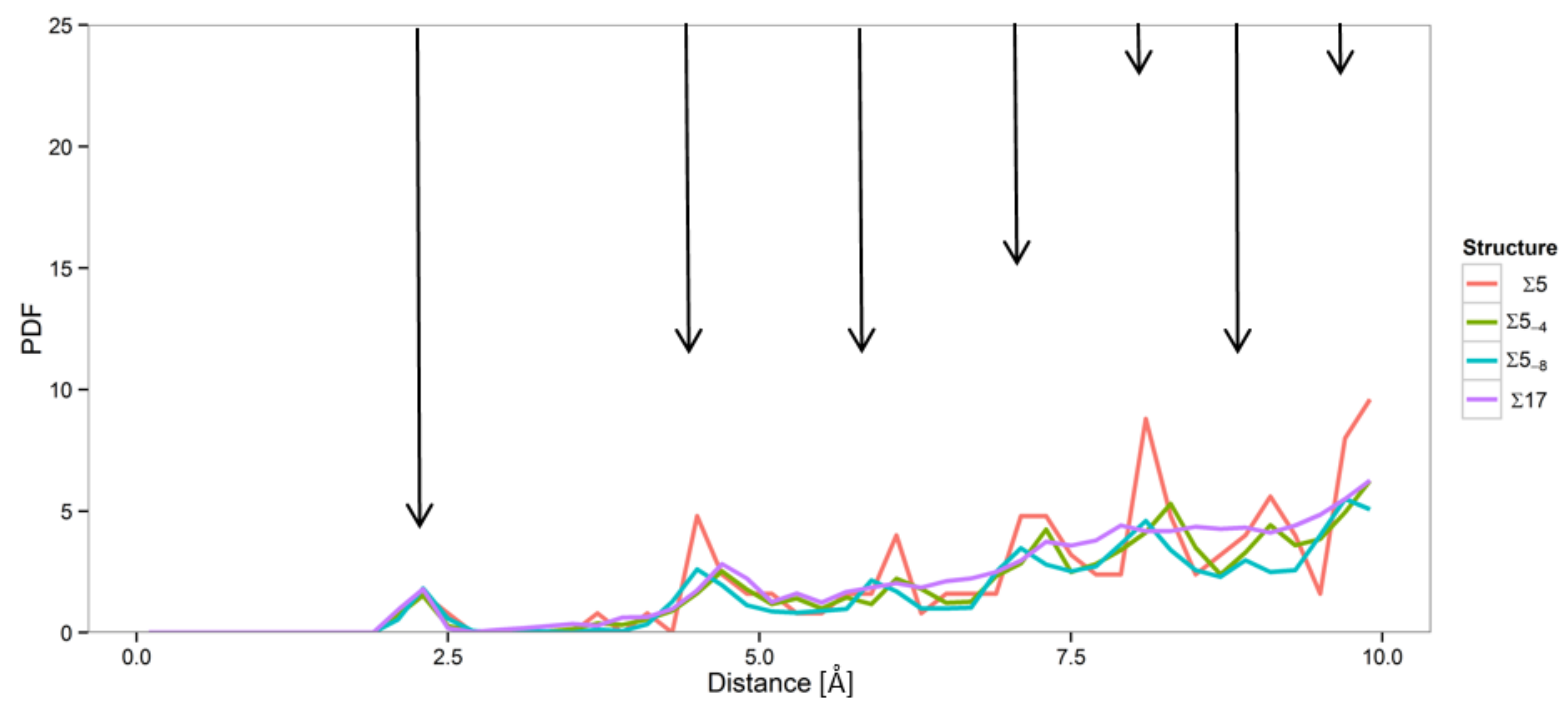

Figure 7 Inter-layer PDF analysis of grain boundaries and bulk. The arrow indicates the peaks in the perfect crystal structure.

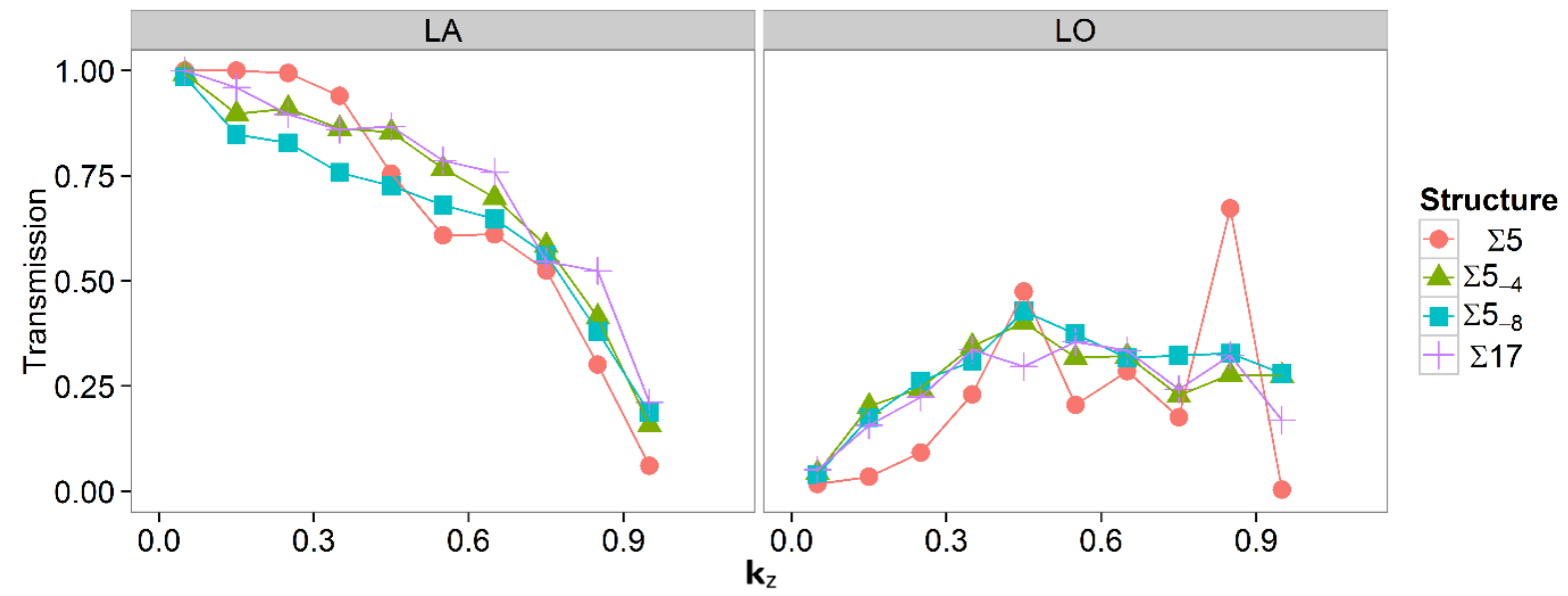

Figure 8 Comparison of the LA and LO phonon transmission of different structures.

\section{DISCUSSION AND CONCLUSIONS}

The transmission coefficients of the individual phonon modes demonstrated complex behavior as a function of the phonon crystal momentum. While low frequency acoustic mode behavior is consistent with the AMM, high frequency modes show strong dependency on the phonon wave vector and on the structure of the GB. However, introduction of additional disorder to the $\Sigma 5$ interface smooths out the variability with regards to wave vector and almost eliminates the differences in the transmission coefficients between different GBs. This 
observation suggests that in the case of non-atomically sharp GBs, the Kapitza conductance should be a very weak function of the misorientation of the grains. The larger the disorder at the grain boundary, the more uniform interface conductance is expected to be. This in turn indicates that experimental results on the polycrystalline samples [28] elucidate an actual Kapitza resistance of the grain boundaries, rather than the average value, since in many of the materials studied GBs are not expected to be sharp in the atomic structure.

In summary, we have studied the detailed phonon scattering at $\mathrm{UO}_{2}$ grain boundary using phonon wave-packet dynamics method. We have calculated the phonon transmission coefficient of both normal and non-normal incident phonons and found the phonon transmission of the latter case decrease significantly. We have also tried to quantify how the detailed atomic structure of the grain boundary affect the phonon transmission. That is, the roughness at the grain boundary actually facilitates certain phonon transmission by smoothing the acoustic impedance of the grain boundary region. By better quantifying the interfacial roughness, it might provide better parameter sets for larger scale models on thermal transport. Finally, we note the limitation of the PWD method, that the anharmonic effects are not captured.

\section{ACKNOWLEDGMENTS}

This work was co-authored by subcontractors (BD, AC and SRP) of the U.S. Government under DOE Contract No. DE-AC07-05ID14517, under the Energy Frontier Research Center (Office of Science, Office of Basic Energy Science, FWP 1356). Accordingly, the U.S. Government retains and the publisher (by accepting the article for publication) acknowledges that the U.S. Government retains a nonexclusive, paid-up, irrevocable, world-wide license to publish or reproduce the published form of this manuscript, or allow others to do so, for U.S. Government purposes. 


\section{REFERENCES}

[1] D.P.H. Hasselman, L.F. Johnson, J. Compos. Mater. 21 (1987) 508.

[2] H. Zhan, Y. Zhang, J.M. Bell, Y.-W. Mai, Y. Gu, Carbon N. Y. 77 (2014) 416.

[3] M.E. Siemens, Q. Li, R. Yang, K.A. Nelson, E.H. Anderson, M.M. Murnane, H.C. Kapteyn, Nat Mater 9 (2010) 26.

[4] E. Swartz, R. Pohl, Rev. Mod. Phys. 61 (1989) 605.

[5] P.E. Hopkins, ISRN Mech. Eng. 2013 (2013) 1.

[6] B. Deng, M. Khafizov, D.H. Hurley, S.R. Phillpot, J. Appl. Phys. 115 (2014) 084910.

[7] P.K. Schelling, S.R. Phillpot, P. Keblinski, Appl. Phys. Lett. 80 (2002) 2484.

[8] T. Watanabe, S. Sinnott, J. Tulenko, R. Grimes, P. Schelling, S. Phillpot, J. Nucl. Mater. 375 (2008) 388.

[9] T. Chen, D. Chen, B.H. Sencer, L. Shao, J. Nucl. Mater. 452 (2014) 364.

[10] P.K. Schelling, S.R. Phillpot, P. Keblinski, J. Appl. Phys. 95 (2004) 6082.

[11] C. Kimmer, S. Aubry, A. Skye, P. Schelling, Phys. Rev. B 75 (2007) 144105.

[12] P. V. Nerikar, K. Rudman, T.G. Desai, D. Byler, C. Unal, K.J. McClellan, S.R. Phillpot, S.B. Sinnott, P. Peralta, B.P. Uberuaga, C.R. Stanek, J. Am. Ceram. Soc. 94 (2011) 1893.

[13] G. Busker, Ph.D. Dissertation, Imperial College of Science, Technology and Medicine, 2002.

[14] K. Govers, S. Lemehov, M. Hou, M. Verwerft, J. Nucl. Mater. 376 (2008) 66.

[15] K. Govers, S. Lemehov, M. Hou, M. Verwerft, J. Nucl. Mater. 366 (2007) 161.

[16] T. Watanabe, S.G. Srivilliputhur, P.K. Schelling, J.S. Tulenko, S.B. Sinnott, S.R. Phillpot, J. Am. Ceram. Soc. 92 (2009) 850.

[17] B. Deng, A. Chernatynskiy, P. Shukla, S.B. Sinnott, S.R. Phillpot, J. Nucl. Mater. 434 (2013) 203.

[18] P. V Nerikar, D.C. Parfitt, L.A. Casillas Trujillo, D.A. Andersson, C. Unal, S.B. Sinnott, R.W. Grimes, B.P. Uberuaga, C.R. Stanek, Phys. Rev. B 84 (2011) 174105.

[19] P. V. Nerikar, Ph.D. Dissertation, University of Florida, 2009.

[20] C.H. Baker, D. a. Jordan, P.M. Norris, Phys. Rev. B 86 (2012) 104306.

[21] Z.T. Tian, B.E. White, Y. Sun, Appl. Phys. Lett. 96 (2010) 3.

[22] N.W. Ashcroft, N.D. Mermin, Solid State Physics, Holt, Rinehart and Winston, 1976.

[23] L. Sun, J.Y. Murthy, 2008 11th Intersoc. Conf. Therm. Thermomechanical Phenom. Electron. Syst. (2008) 1078. 
[24] X. Gu, X. Li, R. Yang, Phys. Rev. B 91 (2015) 205313.

[25] H. Zhao, J.B. Freund, J. Appl. Phys. 97 (2005) 024903.

[26] Z. Zheng, X. Chen, B. Deng, A. Chernatynskiy, S. Yang, L. Xiong, Y. Chen, J. Appl. Phys. 116 (2014).

[27] Z. Tian, K. Esfarjani, G. Chen, Phys. Rev. B 86 (2012) 235304.

[28] A.M. Limarga, D.R. Clarke, Appl. Phys. Lett. 98 (2011) 211906. 